\title{
Multiple Endocrine Neoplasia Type 1: A Case Report With Review of Imaging Findings
}

\author{
Hilary R. Keller, MD, ${ }^{1}$ Jessica L. Record, MD, ${ }^{2}$ Neil U. Lall, $M^{2}$ \\ ${ }^{1}$ Department of Surgery, Guthrie Robert Packer Hospital, Sayre, PA ${ }^{2}$ Department of Radiology, Ochsner Clinic Foundation, New Orleans, LA
}

Background: Multiple endocrine neoplasia type 1 (MEN1) is a rare, autosomal dominant inherited syndrome caused by mutations in the MEN1 tumor suppressor gene. The diagnosis is defined clinically by the presence of 2 or more primary MEN1 tumors (parathyroid, anterior pituitary, and pancreatic islet). We describe the case of a patient who presented with classic history and imaging findings for MEN1.

Case Report: A male in his early thirties with a history of hyperparathyroidism and a transsphenoidal prolactinoma resection presented years later with abdominal symptoms concerning for Zollinger-Ellison syndrome: worsening epigastric abdominal pain, nausea, vomiting, and diarrhea. Contrast-enhanced computed tomography $(C T)$ of the abdomen revealed hyperenhancing pancreatic lesions and duodenal inflammation, suggesting pancreatic neuroendocrine tumor (gastrinoma) with secondary duodenitis. Bilateral indeterminate hypoattenuating adrenal nodules were also seen on contrast-enhanced $\mathrm{CT}$, and follow-up magnetic resonance imaging confirmed benign adrenal adenomas. Furthermore, thyroid ultrasound and sestamibi scintigraphy revealed a parathyroid adenoma. With confirmatory imaging findings, history, and presenting symptoms, the patient was clinically diagnosed with MEN1 syndrome and underwent surgical and medical management.

Conclusion: This case exhibits the classic history with corresponding imaging findings of MEN1 syndrome, including pancreatic neuroendocrine tumors, parathyroid adenoma, and adrenal adenomas. High clinical suspicion for MEN1 should lead to endocrinology evaluation with appropriate laboratory workup and targeted imaging evaluation of the typical endocrine organs as described for this patient.

Keywords: Adrenal gland neoplasms, gastrinoma, multiple endocrine neoplasia type 1, neuroendocrine tumors, parathyroid neoplasms, pituitary neoplasms, Zollinger-Ellison syndrome

Address correspondence to Neil U. Lall, MD, Department of Radiology, Ochsner Clinic Foundation, 1514 Jefferson Hwy., New Orleans, LA 70121. Tel: (504) 842-4796. Email: neil.lall@ochsner.org

\section{INTRODUCTION}

Multiple endocrine neoplasia type 1 (MEN1) is a rare, autosomal dominant inherited syndrome caused by mutations in the MEN1 tumor suppressor gene. The diagnosis is defined clinically by the presence of at least 2 primary MEN1 tumors (parathyroid, anterior pituitary, and pancreatic islet). ${ }^{1}$ We describe the case of a patient with all 3 primary MEN1 tumors with classic imaging findings.

\section{CASE REPORT}

A male in his early thirties presented to the emergency department (ED) after 3 weeks of worsening burning epigastric abdominal pain, nausea, vomiting, and diarrhea. Review of his history revealed a pituitary prolactinoma that had initially presented with headache, blurry vision, and spontaneous cerebrospinal fluid rhinorrhea when the patient was in his late twenties. Magnetic resonance imaging (MRI) had confirmed the diagnosis of pituitary prolactinoma. The patient had undergone subtotal transsphenoidal resection at an out- side hospital and had since been on 0.25 -mg oral cabergoline twice weekly. Additionally, he had had 2 episodes of calcium-rich nephrolithiasis in his late twenties, and during the course of his outpatient endocrinology workup, he was diagnosed with both hypercalcemia and hyperparathyroidism. Family history was positive for a pituitary tumor in his paternal grandmother and a thymic tumor in a paternal aunt. The patient's father died of unknown causes. Although the patient was previously on a proton pump inhibitor (PPI) for severe reflux, this medication had been stopped approximately 3 weeks prior to presentation to accurately evaluate serum gastrin levels because of suspicion of gastrinoma in the setting of possible MEN1 syndrome. The patient's abdominal symptoms became so severe that he presented to the ED prior to follow-up with endocrinology.

During admission to the ED, the patient was evaluated with contrast-enhanced computed tomography (CT) of the abdomen and pelvis that demonstrated duodenal inflammatory changes concerning for duodenitis (Figure $1 \mathrm{~A}$ ). 

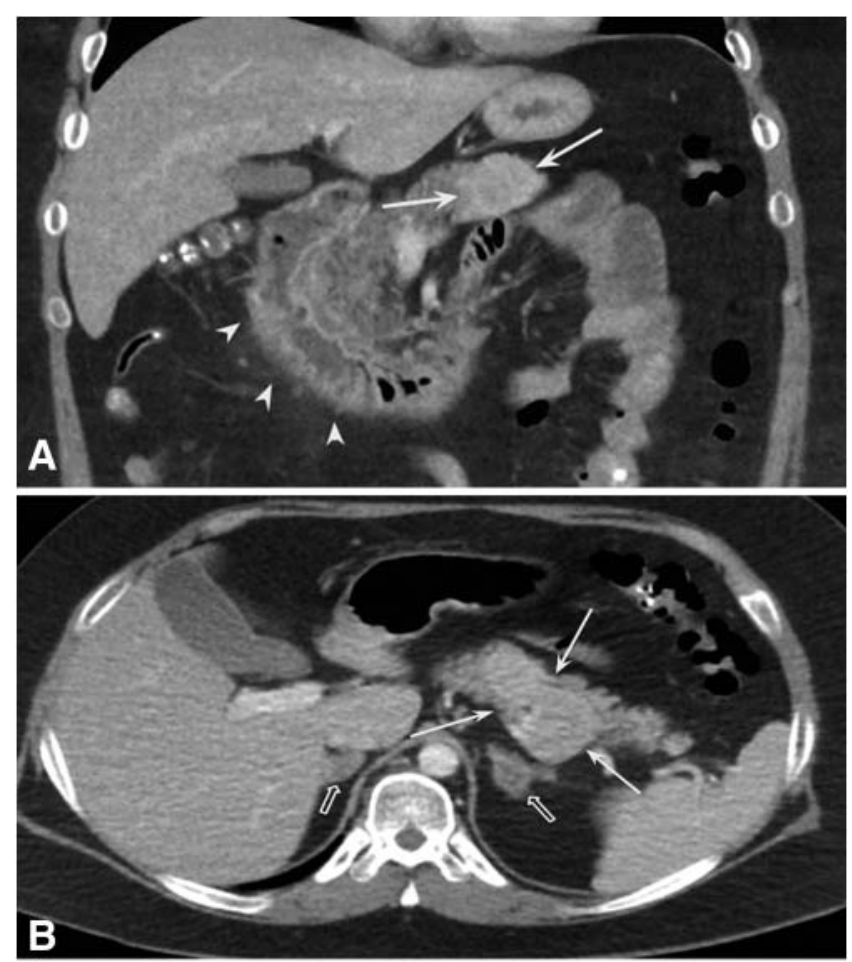

Figure 1. A: Coronal contrast-enhanced computed tomography (CT) of the abdomen demonstrates a large hyperenhancing pancreatic body mass (arrows) and duodenal wall thickening with surrounding fat stranding (arrowheads). B: Axial contrast-enhanced CT of the abdomen shows the hyperenhancing pancreatic body mass (arrows) as well as indeterminate bilateral adrenal nodules (open arrows).

Additionally, 2 hyperenhancing pancreatic masses concerning for neuroendocrine tumors were identified that measured $7.2 \times 4.5 \mathrm{~cm}$ and $1.3 \times 1.4 \mathrm{~cm}$. The larger of the 2 masses was centered in the pancreatic body (Figures $1 \mathrm{~A}$ and 1B). Given concurrent duodenitis, gastrinoma with resultant peptic ulcer disease was suspected. These findings in combination with the history of pituitary prolactinoma and hyperparathyroidism suggested MEN1 syndrome as had been clinically suspected. Additionally, bilateral indeterminate hypoattenuating adrenal nodules were identified on contrast-enhanced CT (Figure 1B).

The patient was admitted for further workup. Serum gastrin level, which was markedly elevated at $758 \mathrm{pg} / \mathrm{mL}$ (reference range, $0.0-110.0 \mathrm{pg} / \mathrm{mL}$ ), was obtained prior to resuming PPI therapy. Esophagogastroduodenoscopy demonstrated esophagitis, gastritis, and multiple nonbleeding duodenal ulcers. Endoscopic ultrasound confirmed the large $(4.2 \times 2.7 \times 2.8 \mathrm{~cm})$ pancreatic body mass (Figure 2$)$; biopsy revealed a well-differentiated neuroendocrine tumor compatible with a gastrinoma. The patient's symptoms were controlled with resumption of PPI therapy, and he was subsequently discharged on twice daily PPI therapy. Evaluation for metastatic disease was performed with somatostatin scintigraphy; however, the patient's known multiple pancreatic neuroendocrine tumors were not positive on this examination, limiting its utility. The patient eventually underwent distal pancreatectomy with lymph node resection. Pathology confirmed 2 well-differentiated neuroendocrine tumors corresponding to the masses identified on contrastenhanced CT. The largest tumor was classified as intermediate grade (World Health Organization grade 2) and measured at least $6.6 \mathrm{~cm}$; a single metastatic inferior pancreaticoduodenal lymph node was identified.

Further workup of the bilateral adrenal lesions identified on contrast-enhanced CT was performed with MRI of the abdomen. These nodules demonstrated diffuse loss of signal on opposed-phase abdominal MRI (Figure 3), diagnostic of benign adenomas. The patient's cortisol tests were within normal limits, suggesting nonfunctioning adenomas. Plasma metanephrine and normetanephrine, obtained to definitively rule out pheochromocytoma prior to surgery, were within normal limits.

The patient's serum calcium was elevated at $12.7 \mathrm{mg} / \mathrm{dL}$ (reference range, $8.7-10.5 \mathrm{mg} / \mathrm{dL}$ ), and his parathyroid hormone level was elevated at $259 \mathrm{pg} / \mathrm{mL}$ (reference range, 9.0-77 pg/mL). Ultrasound to evaluate for possible parathyroid adenoma revealed a hypoechoic lesion inferior to the right thyroid lobe (Figure 4A). Technetium Tc-99m sestamibi parathyroid scintigraphy revealed delayed washout of radiotracer near the lower pole of the right thyroid lobe corresponding to the lesion found on ultrasound and compatible with parathyroid adenoma (Figures $4 \mathrm{~B}$ and $4 \mathrm{C}$ ). The patient underwent subtotal parathyroidectomy with removal of 3.5 of the 4 glands. Pathology confirmed an adenoma within the right lower gland and hyperplasia of the remainder of the resected glands. The patient's parathyroid hormone level normalized to $24 \mathrm{pg} / \mathrm{mL}$ immediately after removal of the glands.

Because the patient had a regional nodal metastasis from his gastrinoma, he is being treated with monthly lanreotide infusions and will undergo periodic imaging surveillance. $\mathrm{He}$ is maintained on daily cabergoline therapy for his residual prolactinoma and will be followed with periodic MRI and prolactin level surveillance. No dedicated follow-up of the adrenal adenomas is required because they are nonfunctioning.

\section{DISCUSSION}

MEN1 syndrome is most commonly inherited as an autosomal dominant condition (90\%) but can occur sporadically, with an incidence of $0.25 \%$ from random postmortem studies. $^{1,2}$ The diagnosis of MEN1 can be made clinically, on the basis of family history, or directly with genetic testing for a MEN1 mutation. ${ }^{1}$ The clinical diagnosis of MEN1 includes the presence of 2 or more primary endocrine tumors associated with MEN1 (parathyroid adenomas, enteropancreatic tumors, and pituitary adenomas)..$^{1-4}$ The diagnosis on the basis of family history requires only 1 of the associated primary endocrine tumors in someone who has a first-degree relative with diagnosed MEN1. ${ }^{1}$ Our patient had a history of a pituitary prolactinoma and newly discovered pancreatic and parathyroid tumors, meeting the clinical criteria for diagnosis. His young age at presentation and family history of neuroendocrine tumors on his father's side suggest familial MEN1; however, the diagnostic criteria only include first-degree relatives. ${ }^{1}$

Parathyroid adenomas are the most frequently occurring tumor in patients with MEN1, occurring in $90 \%$ of patients by age $40 .^{2,5}$ These tumors can cause primary hyperparathyroidism, and patients may present with hypercalcemia, 


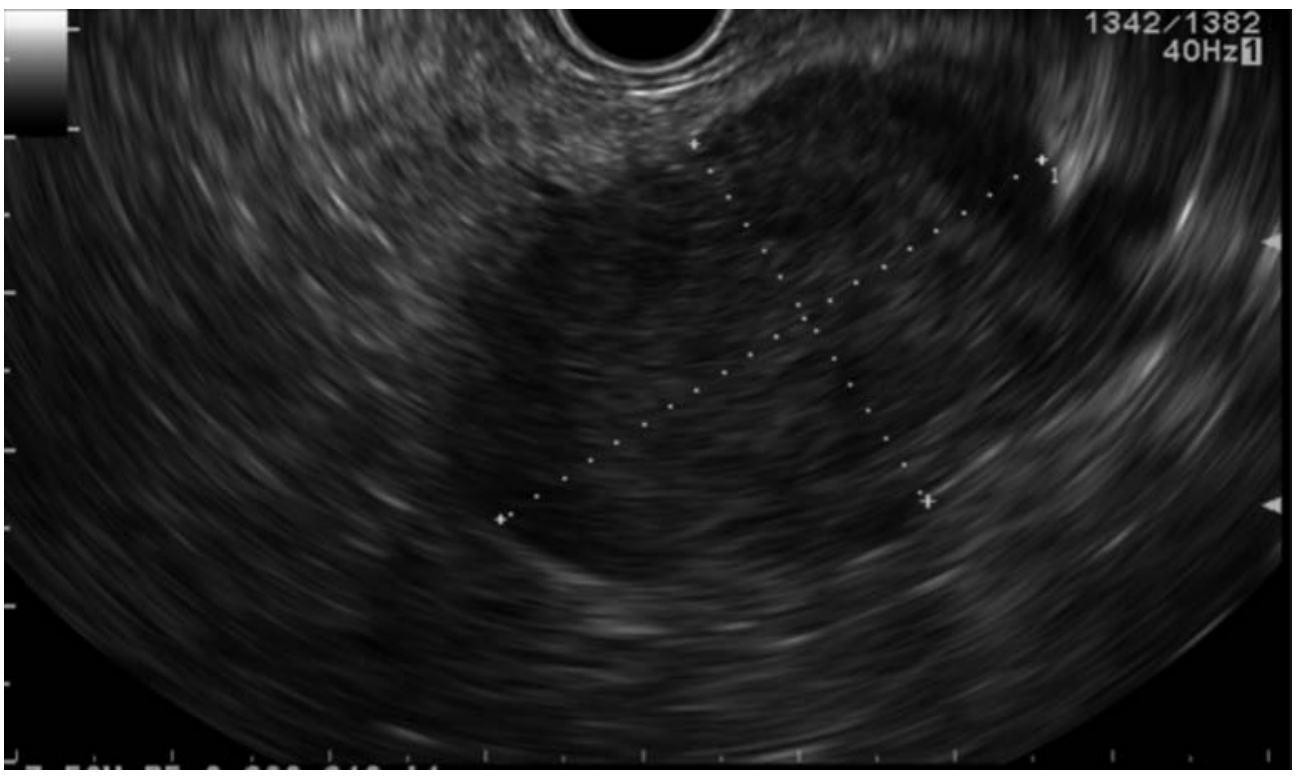

Figure 2. Endoscopic ultrasound shows the corresponding $4.2 \times 2.7 \times 2.8-\mathrm{cm}$ heterogeneously echogenic mass at the pancreatic body that was biopsied.

nephrolithiasis, and osteitis fibrosa cystica. ${ }^{1,3,4}$ The age of onset of symptoms of parathyroid adenoma is earlier in patients with MEN1 than in those without, typically occurring at approximately 20-25 years of age compared to 55 years of age, respectively. ${ }^{1}$ Our patient presented in his early thirties, but he had previously experienced 2 episodes of nephrolithiasis during his late twenties with calcium-rich stones. During workup in the ED, the patient's serum calcium level and parathyroid hormone were both elevated.

Evaluation for patients with hyperparathyroidism typically includes an ultrasound as an initial screening for underlying parathyroid adenoma followed by nuclear technetium Tc99m sestamibi scintigraphy for confirmation. Sestamibi scintigraphy has a sensitivity and specificity of $>90 \%$ for lesions that demonstrate retention of sestamibi radiotracer on delayed imaging. ${ }^{6}$
Management of parathyroid adenomas is typically surgical, with removal of the overactive glands with either a subtotal or total parathyroidectomy; however, noting that patients with MEN1 have an increased risk of persistent or recurrent hypercalcemia after subtotal surgery, which is thought to be secondary to their tendency toward multiglandular disease, is important. ${ }^{1,7}$ In our case, one parathyroid adenoma was identified by initial ultrasound screening and confirmed with sestamibi scintigraphy. The patient subsequently underwent subtotal parathyroidectomy with confirmatory pathology. Biochemical screening for primary hyperparathyroidism in patients with MEN1 should be performed annually with assessment of serum calcium and parathyroid hormone levels. ${ }^{1}$

Although not the most common tumors, malignant enteropancreatic neuroendocrine tumors are the leading cause of


Figure 3. Axial magnetic resonance imaging with in-phase (A) and opposed-phase (B) imaging through the adrenal glands demonstrates diffuse loss of signal within the adrenal nodules on opposed-phase imaging, diagnostic of benign adenomas (arrows). A lobular pancreatic body mass is noted (arrowheads). 


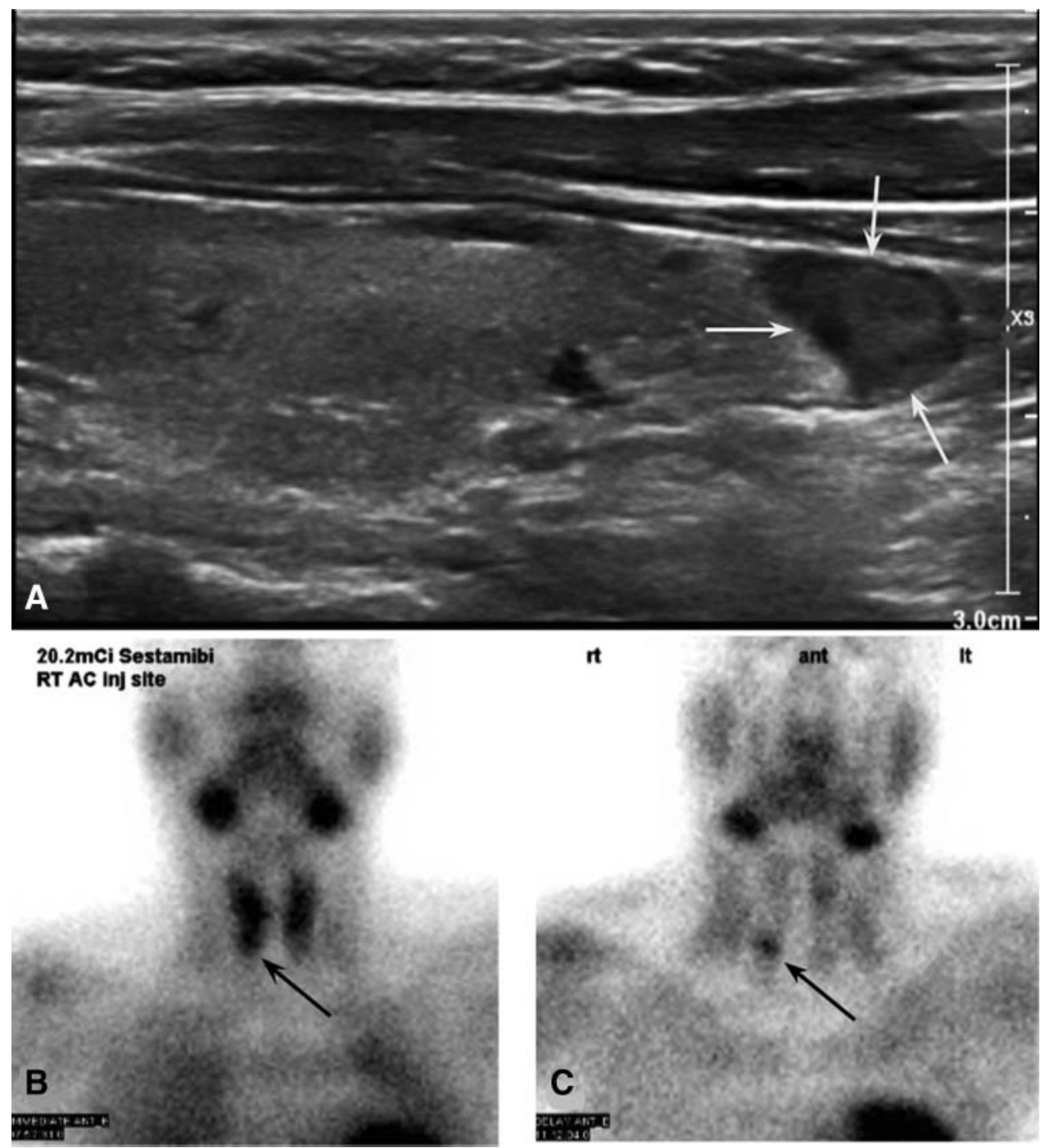

Figure 4. Longitudinal ultrasound (A) through the right lobe of the thyroid gland demonstrates a hypoechoic nodule inferior to the right lobe (white arrows). Immediate (B) and delayed (C) sestamibi scans were obtained for further characterization. Focally increased uptake with a delayed washout is visible along the lower pole of the right thyroid lobe (black arrows), confirming that the nodule seen on ultrasound was a parathyroid adenoma.

mortality in patients with MEN1. ${ }^{5}$ Associated tumors of the pancreatic islet cells can be either functioning (hormone secreting) or nonfunctioning (non-hormone secreting). Hormone-secreting tumors include gastrinomas (40\% incidence), insulinomas (10\% incidence), and vipomas and glucagonomas $(2 \%$ incidence $){ }^{2}$ Gastrinomas, the most commonly occurring enteropancreatic tumor in patients with MEN1, are often multiple, with metastases identified in approximately $50 \%$ of these patients at the time of diagnosis. $^{2}$ Malignant gastrinomas account for the majority of neuroendocrine tumor-related MEN1 deaths. ${ }^{5}$ Clinically, gastrinomas cause gastric acid hypersecretion and recurrent peptic ulcers with diarrhea, known as Zollinger-Ellison syndrome (ZES). The symptoms of ZES can be controlled medically with PPIs, and abrupt discontinuation of PPI therapy to evaluate serum gastrin levels may drastically exacerbate symptoms, as was seen in our patient. ${ }^{8,9}$ Death related to gastric acid hypersecretion is uncommon since the advent of PPIs. ${ }^{5}$

Pancreatic neuroendocrine tumors are best visualized in arterial phase contrast-enhanced CT as well-circumscribed hyperenhancing masses that are less conspicuous during the venous phase in contradistinction to pancreatic ductal carcinomas that typically have a hypoenhancing appearance. Our patient had 2 hyperenhancing pancreatic masses that were pathologically proven to be well-differentiated neuroendocrine tumors and had clinical evidence of gastrin hypersecretion.

Somatostatin receptor scintigraphy is often beneficial in identifying smaller lesions or metastases and has a higher sensitivity and specificity than CT or MRI, particularly for extrahepatic gastrinomas. ${ }^{10-12}$ The somatostatin analog used, octreotide, binds to only 2 of the 6 subtypes of somatostatin receptors (types 2 and 5). However, because $80 \%$ of 
enteropancreatic neuroendocrine tumors express somatostatin receptor subtype 2 , the sensitivity for detecting these tumors is typically good. ${ }^{13}$ For gastrinomas, the overall sensitivity of somatostatin scintigraphy varies from $60 \%$ $90 \% .^{13,14}$ Our patient's gastrinoma did not demonstrate uptake on initial somatostatin receptor scintigraphy, presumably because his particular tumor did not express 1 of these 2 somatostatin receptor subtypes, limiting the utility of this examination for evaluation of metastases and followup.

While symptoms of hypergastrinemia can be controlled medically, definitive treatment of these tumors with surgical resection and regional lymphadenectomy is desired because of their propensity to metastasize. ${ }^{10,15-17}$ Additionally, 2014 data have shown that treatment with lanreotide, a somatostatin analog, can prolong progression-free survival in patients with grade 1 and grade 2 metastatic enteropancreatic neuroendocrine tumors. ${ }^{18}$ Because our patient was found to have a metastatic lymph node during surgery, he is being treated with monthly lanreotide infusions. In patients with known MEN1, biochemical screening for enteropancreatic neuroendocrine tumors should be performed annually with a fasting gastrointestinal hormone profile (including gastrin, glucagon, and vasointestinal polypeptide). Radiologic screening of the pancreas and duodenum with $\mathrm{CT}$, $\mathrm{MRI}$, or endoscopic ultrasound should be performed at least annually, although consensus on optimal screening has not been reached. ${ }^{1}$

Pituitary tumors may occur in up to $60 \%$ of patients with MEN1, although the frequency reported in the literature is variable. $^{2}$ Prolactinomas are the most commonly occurring pituitary tumors in patients with MEN1, with a prevalence of $20 \%$ by age 40 years. $^{2}$ Less common pituitary tumors include somatotropin-producing adenomas, corticotropin adenomas, and nonfunctioning tumors. ${ }^{1}$ Patients with pituitary tumors can present with headaches and visual field deficits because of tumor size and growth. Prolactinomas in particular can cause erectile dysfunction and decreased libido in men or amenorrhea and galactorrhea in women resulting from increased prolactin. ${ }^{1}$ The management of prolactinomas typically involves transsphenoidal resection of the tumor or medical management with bromocriptine or cabergoline therapy. MEN1-associated pituitary tumors, however, tend to be more aggressive and resistant to therapy and surgery than sporadic pituitary tumors. ${ }^{1,19,20}$ Adequate follow-up is therefore necessary to track the onset of symptoms in such patients.

Our patient initially presented with symptoms of mass effect (headache and visual changes) as well as the rare complication of cerebrospinal fluid rhinorrhea. In patients with MEN1, annual biochemical screening for pituitary tumors is advised (serum prolactin and insulin-like growth factor 1 levels), as well as imaging surveillance with MRI of the pituitary gland every 3-5 years. ${ }^{1}$

Although not a defining feature of MEN1, adrenal tumors have been reported in $20 \%-40 \%$ of patients with MEN1 and are typically benign and nonfunctional. ${ }^{2}$ However, pheochromocytomas and adrenocortical carcinomas have also been reported. ${ }^{21}$ In our patient, bilateral adrenal adenomas were diagnosed on MRI and determined to be nonfunctioning based on cortisol levels. Additionally, pheochromocytoma was definitely ruled out with laboratory work. Screening for adrenal lesions in patients with MEN1 should be performed with abdominal imaging (MRI or CT) every 3 years. If a lesion is identified, it should be assessed for malignant features with continued imaging surveillance, although a specific time interval for this follow-up has not been established. Biochemical evaluation with assessment of aldosterone and cortisol levels should only be performed if clinical features are present or if the lesion is $>1 \mathrm{~cm} .^{1}$

The life expectancy of patients with MEN1 is less than that of the average population, with a mean age of death of 55 years. ${ }^{5}$ These patients should be managed by a multidisciplinary team with expertise in neuroendocrine tumors and should receive appropriate biochemical and radiologic surveillance. ${ }^{1}$

\section{CONCLUSION}

Our patient demonstrates a classic example of MEN1 syndrome with tumors in all 3 defining endocrine organs, including pituitary prolactinoma, parathyroid adenoma, and pancreatic neuroendocrine tumors (gastrinomas). When the clinical suspicion for MEN1 is high, endocrinology evaluation with appropriate laboratory workup and targeted imaging evaluation of the typical endocrine organs as described for this patient are advised with careful multidisciplinary management and follow-up.

\section{ACKNOWLEDGMENTS}

The authors have no financial or proprietary interest in the subject matter of this article.

\section{REFERENCES}

1. Thakker RV, Newey PJ, Walls GV, et al; Endocrine Society. Clinical practice guidelines for multiple endocrine neoplasia type 1 (MEN1). J Clin Endocrinol Metab. 2012 Sep;97(9):29903011. doi: 10.1210/jc.2012-1230.

2. Almeida MQ, Stratakis CA. Solid tumors associated with multiple endocrine neoplasias. Cancer Genet Cytogenet. 2010 Nov;203(1):30-36. doi: 10.1016/j.cancergencyto.2010.09.006.

3. Donegan D, Singh Ospina N, Rodriguez-Gutierrez R, et al. Longterm outcomes in patients with multiple endocrine neoplasia type 1 and pancreaticoduodenal neuroendocrine tumours. Clin Endocrinol (Oxf). 2017 Feb;86(2):199-206. doi: 10.1111/ cen.13264.

4. Thakker RV. Multiple endocrine neoplasia type 1 (MEN1) and type 4 (MEN4). Mol Cell Endocrinol. 2014 Apr 5;386(1-2):2-15. doi: 10.1016/j.mce.2013.08.002.

5. Ito $T$, Igarashi $H$, Uehara $H$, Berna MJ, Jensen RT. Causes of death and prognostic factors in multiple endocrine neoplasia type 1: a prospective study: comparison of 106 MEN1/

Zollinger-Ellison syndrome patients with 1613 literature MEN1 patients with or without pancreatic endocrine tumors. Medicine (Baltimore). 2013 May;92(3):135-181. doi: 10.1097/ MD.0b013e3182954af1.

6. Michaud L, Balogova S, Burgess A, et al. A pilot comparison of 18F-fluorocholine PET/CT, ultrasonography and 1231/99mTcsestaMIBI dual-phase dual-isotope scintigraphy in the preoperative localization of hyperfunctioning parathyroid glands in primary or secondary hyperparathyroidism: influence of thyroid anomalies. Medicine (Baltimore). 2015 Oct;94(41): e1701. doi: 10.1097/MD.0000000000001701.

7. Doherty GM, Lairmore TC, DeBenedetti MK. Multiple endocrine neoplasia type 1 parathyroid adenoma development over time. World J Surg. 2004 Nov;28(11):1139-1142. 
8. Singh Ospina N, Donegan D, Rodriguez-Gutierrez R, Al-Hilli Z, Young WF Jr. Assessing for multiple endocrine neoplasia type 1 in patients evaluated for Zollinger-Ellison syndrome-clues to a safer diagnostic process. Am J Med. 2017 May;130(5):603-605. doi: 10.1016/j.amjmed.2016.11.035.

9. Poitras P, Gingras MH, Rehfeld JF. The Zollinger-Ellison syndrome: dangers and consequences of interrupting antisecretory treatment. Clin Gastroenterol Hepatol. 2012 Feb;10 (2):199-202. doi: 10.1016/j.cgh.2011.08.012.

10. Epelboym I, Mazeh H. Zollinger-Ellison syndrome: classical considerations and current controversies. Oncologist. 2014 Jan;19(1):44-50. doi: 10.1634/theoncologist.2013-0369.

11. Ito T, Jensen RT. Molecular imaging in neuroendocrine tumors: recent advances, controversies, unresolved issues, and roles in management. Curr Opin Endocrinol Diabetes Obes. 2017 Feb;24 (1):15-24. doi: 10.1097/MED.0000000000000300.

12. Delle Fave G, O'Toole D, Sundin A, et al; Vienna Consensus Conference participants. ENETS Consensus Guidelines Update for Gastroduodenal Neuroendocrine Neoplasms. Neuroendocrinology. 2016;103(2):119-124. doi: 10.1159/ 000443168.

13. Intenzo CM, Jabbour S, Lin $\mathrm{HC}$, et al. Scintigraphic imaging of body neuroendocrine tumors. Radiographics. 2007 Sep-Oct;27 (5):1355-1369.

14. Kwekkeboom D, Krenning EP, de Jong M. Peptide receptor imaging and therapy. J Nucl Med. 2000 Oct;41(10):1704-1713.
15. Hashim YM, Trinkaus KM, Linehan DC, et al. Regional lymphadenectomy is indicated in the surgical treatment of pancreatic neuroendocrine tumors (PNETs). Ann Surg. 2014 Feb;259(2):197-203. doi: 10.1097/SLA.0000000000000348.

16. Burns WR, Edil BH. Neuroendocrine pancreatic tumors: guidelines for management and update. Curr Treat Options Oncol. 2012 Mar;13(1):24-34. doi: 10.1007/s11864-011-0172-2.

17. Doi R. Determinants of surgical resection for pancreatic neuroendocrine tumors. J Hepatobiliary Pancreat Sci. 2015 Aug;22(8):610-617. doi: 10.1002/jhbp.224.

18. Caplin ME, Pavel M, Ćwikła JB, et al; CLARINET Investigators. Lanreotide in metastatic enteropancreatic neuroendocrine tumors. N Engl J Med. 2014 Jul 17;371(3):224-233. doi: 10.1056/ NEJMoa1316158.

19. Trouillas J, Labat-Moleur F, Sturm N, et al; Groupe d'études des Tumeurs Endocrines. Pituitary tumors and hyperplasia in multiple endocrine neoplasia type 1 syndrome (MEN1): a casecontrol study in a series of 77 patients versus 2509 non-MEN1 patients. Am J Surg Pathol. 2008 Apr;32(4):534-543. doi: 10.1097/PAS.0b013e31815ade45.

20. Beckers A, Betea D, Valdes Socin H, Stevenaert A. The treatment of sporadic versus MEN1-related pituitary adenomas. $J$ Intern Med. 2003 Jun;253(6):599-605.

21. Langer $P$, Cupisti K, Bartsch DK, et al. Adrenal involvement in multiple endocrine neoplasia type 1. World J Surg. 2002 Aug;26 (8):891-896.

This article meets the Accreditation Council for Graduate Medical Education and the American Board of Medical Specialties Maintenance of Certification competencies for Patient Care and Medical Knowledge. 